\title{
Systemic Acquired Resistance Induced by Agrobacterium tumefaciens in Peach and Differential Expression of PR1 Genes
}

\author{
Fengge Hao', Lirong Wang, Ke Cao, Xinwei Wang, Weichao Fang, \\ Gengrui Zhu, and Changwen Chen \\ Zhengzhou Fruit Research Institute, Chinese Academy of Agriculture Sciences, \\ Zhengzhou 450009, People's Republic of China
}

Additional index words. systemic acquired resistance, crown gall disease, peach, pathogenesisrelated protein 1 , salicylic acid

\begin{abstract}
Crown gall disease caused by Agrobacterium tumefaciens affects a wide range of horticultural plants, and has no effective treatment. During the evaluation of crown gall resistance of peach germplasm resources, we observed enhanced resistance to subsequent invasion that was activated by virulence of $A$. tumefaciens in two peach cultivars. To further verify the phenotype observed in field experiments, systemic acquired resistance (SAR)-related salicylic acid (SA) and $P R 1$ genes were investigated. The levels of SA were elevated in two cultivars, and these high levels were maintained for 35 days postinoculation. Compared with mock-inoculated controls, eight of the 22 candidate PpPR1 genes in $A$. tumefaciens-inoculated samples were significantly upregulated and three were downregulated in response to inoculation with $A$. tumefaciens. These data suggested that SA-induced SAR was activated in two peach cultivars by virulent $A$. tumefaciens infection. In addition, the eight induced $P p P R 1$ genes can be used as molecular markers in defense studies in peach.
\end{abstract}

Plants have evolved an array of strategies to defend themselves against pathogens. The constitutive structural and chemical barriers are the first line of defense. When the pathogens go past these barriers, twobranched inducible defenses are recruited to halt further ingress (Pieterse et al., 2009). Pattern-triggered immunity (PTI) is a nonhost resistance initiated by the recognition of pathogen-associated molecular patterns (Jones and Dangl, 2006; Mishina and Zeier, 2007). For a successful colonization, pathogens deliver effectors into the plant cells to suppress the PTI. In turn, plants develop resistance proteins that recognize these attacker-specific effectors, activating effector-triggered immunity (ETI) (Jones and Dangl, 2006). Effector-triggered immunity is an amplified PTI response, resulting in gene-for-gene resistance and usually a hypersensitive cell death response at the infection site (Jones and Dangl, 2006).

Received for publication 3 Dec. 2014. Accepted for publication 23 Feb. 2015.

This project was supported by the Special Fund for Agro-scientific Research in the Public Interest (No. 20133093) and Agricultural Science and Technology Innovation Project of CAAAS (No. CAAS-ASTIP-2015-ZFRI).

We are grateful to Rongjun Guo and Shifang Li (Institute of Plant Protection, Chinese Academy of Agricultural Sciences) for offering pathogen used in this work.

${ }^{1}$ To whom reprint requests should be addressed; e-mail Wlirong2009@sina.com.
Besides resistance responses at the site of attempted ingress, plants can initiate SAR in tissues distant from the initial infection, which confers stable and broad-spectrum systemic resistance against subsequent attackers (Cameron et al., 1994; Durrant and Dong, 2004). The phytohormone SA is known to participate in regulating SAR response (Loake and Grant, 2007). Once the pathogen is detected, the plant amplifies the production of SA and induces the expression of pathogenesis-related (PR) protein genes, which leads to the establishment of SAR (van Loon and van Strien, 1999). Routinely, the expression of PRl genes and increased levels of SA are used as markers to manifest the SAR induction (Anand et al., 2008; Govrin and Levine, 2002; Wu et al., 2013). Induced systemic resistance (ISR), elicited by plant growth-promoting rhizobacteria, is another form of induced resistance in plants, and phenotypically it is similar to the SAR. Unlike SAR, the ISR is not associated with the expression of $P R$ genes or accumulation of SA (Vallad and Goodman, 2004; van Loon et al., 1998).

Agrobacterium tumefaciens, a soil-borne bacterium, causes formation of crown galls in many plant species. During infection, a specific segment of the tumor-inducing plasmid, the transfer DNA (T-DNA), is transferred from the bacterium and integrated into the plant genome (Lee et al., 2009; Pitzschke and Hirt, 2010). Genes encoded by T-DNA are expressed, resulting in the over production of auxins and cytokinins and leading to an abnormal cell proliferation and tumor formation at the infection site.

Although, the pathogen infection process has been elucidated, little is known about the defense mechanism in host plants. Resistance genes that were screened from the Rhizobium vitis-inoculated cDNA library in grapevine suggest that SA signaling is involved in defense responses (Choi et al., 2010). Previous studies have also demonstrated that SAR retards Agrobacterium infection in Nicotiana benthamiana (Anand et al., 2008). In the course of Arabidopsis-Agrobacterium interaction, the transcript of $P R 1$ gene could not be detected, indicating that SAR was not induced in the host organism (Lee et al., 2009).

Crown gall disease is a serious problem in horticultural crops worldwide, including peach, and using plant resistance remains an alternative approach to combat this disease (Escobar and Dandekar, 2003; Zoina and Raio, 1999). During the evaluation of crown gall resistance in peach wild germplasm resources and cultivars, we observed two peach cultivars that presented enhanced resistance when infested with $A$. tumefaciens. Further investigation indicated that the SA-induced SAR was activated instead of ISR. To our knowledge, this is the first example of SAR activated by $A$. tumefaciens in horticultural crops. In addition, two PRI genes have been isolated from a peach tree infected by Xanthomonas campestris pv. pruni (Sherif et al., 2012), but no data have shown the expression profiles of $P R I$ gene family at the SAR. In this study, we assayed transcript expression of $P R 1$ genes in peach SAR induced by $A$. tumefaciens infection.

\section{Materials and Methods}

Plant material. Plant material used for evaluation of the resistance to crown gall was classified into two classes based on the propagation methods. The first class included 40 accessions or cultivars, most of which were obtained from the wild germplasm resources collection and some cultivars were placed in this class because of the same propagation method (Table 2). They were propagated from seeds and every accession or cultivar was presented with $10-70$ seedlings. The second class was propagated by grafting and contained 189 peach germplasms including Chinese landraces, breed cultivars, and related species (data not shown). Four replications of each germplasm were grafted on the rootstock of Prunus persica. Seedlings and grafted saplings were prepared previously in a fumigated soil and were considered to be intact and transplanted to experimental field in the spring of the following year.

To characterize the enhanced resistance observed in field experiments, two cultivars 'Honggengansutao' and 'Xibei13-1' were used for their inducible resistance, and further investigation proved that they were the most resistant and the most susceptible to crown gall disease, respectively. Fifty 
Table 1. Primers used in qPCR of $P p$ - $P R 1 \mathrm{~s}$ and reference genes.

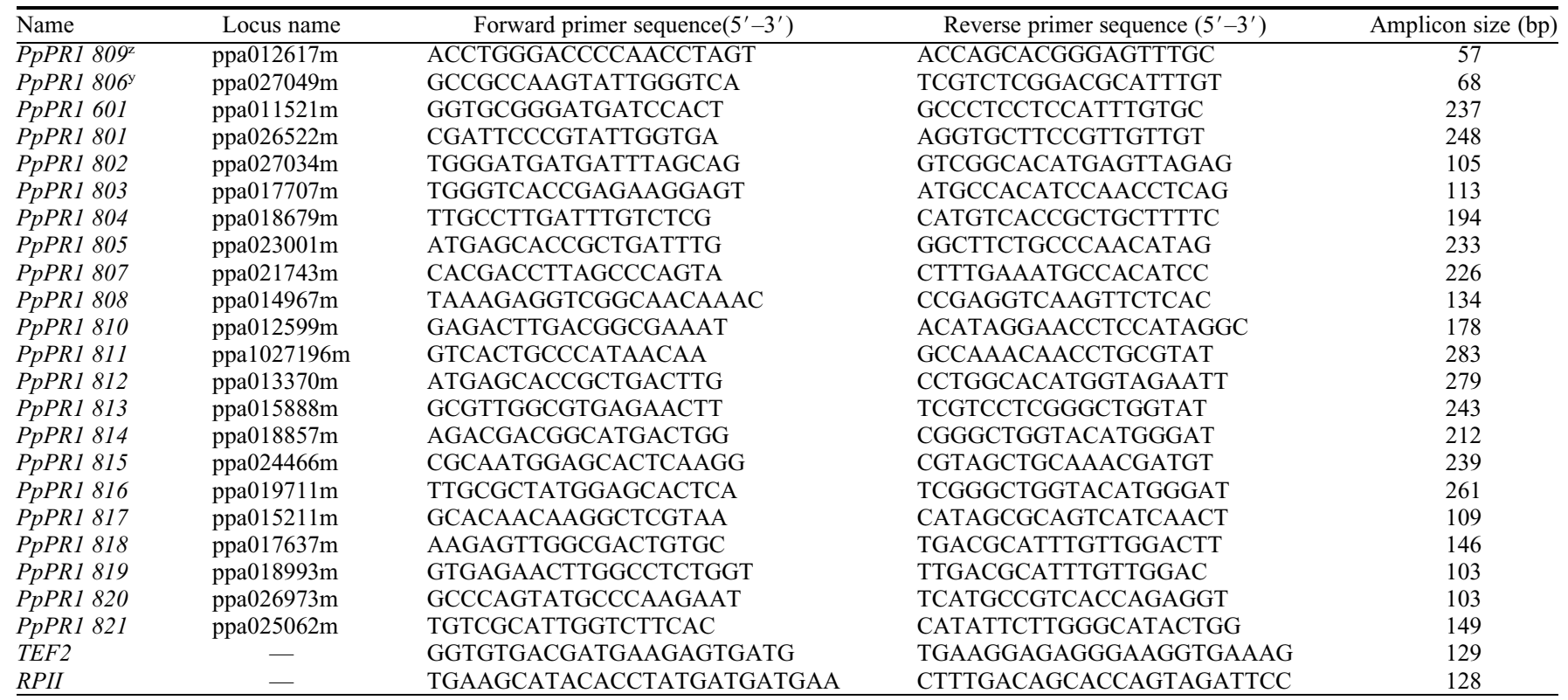

z, yThe two pairs of primers were obtained from Sherif et al. (2012).

Table 2. Frequency of tumor occurrence and tumor size in seven peach accessions/cultivars of seedlings inoculated with virulence strains of Agrobacterium tumefaciens at different time periods.

\begin{tabular}{|c|c|c|c|c|c|c|c|}
\hline \multirow[b]{2}{*}{ Accession/cultivar } & \multirow[b]{2}{*}{ Genetic origin } & \multirow[b]{2}{*}{ Code } & \multicolumn{2}{|c|}{$2013^{x}$} & \multicolumn{3}{|c|}{$2014^{\mathrm{w}}$} \\
\hline & & & $5-23$ & $6-22$ & $5-18$ & $6-07$ & $6-27$ \\
\hline \multirow{2}{*}{ Honggengansutao } & P. kansuensis & $\mathrm{F}(\%)$ & 55.6 & 16.7 & 15.6 & 16.2 & 16.6 \\
\hline & & $\mathrm{D}(\mathrm{mm})$ & $13.22 \pm 4.96$ & $2.22 \pm 2.02$ & $3.42 \pm 3.56$ & $3.80 \pm 3.66$ & $2.67 \pm 2.91$ \\
\hline Xibei 13-1 & & $\mathrm{D}(\mathrm{mm})$ & $11.88 \pm 2.89$ & $9.57 \pm 4.05$ & $9.84 \pm 3.75$ & $10.84 \pm 4.94$ & $8.85 \pm 3.79$ \\
\hline \multirow[t]{2}{*}{ Zhongtaokangzhen $1^{1}$} & P. persica & $\mathrm{F}(\%)$ & 75.2 & 72.0 & 75.5 & 73.8 & 73.2 \\
\hline & & $\mathrm{D}(\mathrm{mm})$ & $14.34 \pm 5.31$ & $14.76 \pm 6.43$ & $13.28 \pm 5.31$ & $13.79 \pm 6.92$ & $11.34 \pm 5.31$ \\
\hline \multirow[t]{2}{*}{ Wanzhouyetao $1^{2}$} & P. persica & $\mathrm{F}(\%)$ & 82.6 & 78.2 & 80.6 & 81.3 & 80.5 \\
\hline & & $\mathrm{D}(\mathrm{mm})$ & $7.31 \pm 3.32$ & $5.28 \pm 3.01$ & $6.47 \pm 2.82$ & $7.22 \pm 4.83$ & $6.86 \pm 3.63$ \\
\hline \multirow[t]{2}{*}{ Rutgers Redleaf $^{3}$} & P. persica & $\mathrm{F}(\%)$ & 85.7 & 79.8 & 81.3 & 82.7 & 83.0 \\
\hline & & $\mathrm{D}(\mathrm{mm})$ & $11.2 \pm 4.83$ & $13.4 \pm 6.64$ & $11.8 \pm 5.74$ & $10.37 \pm 5.78$ & $11.76 \pm 3.88$ \\
\hline Okinawa $^{3}$ & $P$. persica & $\mathrm{F}(\%)$ & 100.0 & 98.2 & 100.0 & 100.0 & 100.0 \\
\hline
\end{tabular}

replications of each cultivar were prepared by grafting on the rootstock of $P$. persica. Dormant 1-year-old saplings were planted in pots, $40 \mathrm{~cm}$ diameter and $50 \mathrm{~cm}$ high, filled with soil mixture (1 part peatsoil:1 part vermiculite: 1 part sand). Shoot tips were removed when the seedlings reached $30 \mathrm{~cm}$ in height to stimulate lateral shoot growth. Four to five shoots prepared for inoculation were retained per sapling.

Bacterial inoculations. Agrobacterium tumefaciens strain AT-4-3 (biovar 2), isolated from a peach tree in Hebei Province, was used for inoculation. Bacteria were cultured in yeast extract and beef extract (YEB) medium $\left(1 \mathrm{~g} \cdot \mathrm{L}^{-1}\right.$ yeast extract, $5 \mathrm{~g} \cdot \mathrm{L}^{-1}$ beef extract, $5 \mathrm{~g} \cdot \mathrm{L}^{-1}$ tryptone, $5 \mathrm{~g} \cdot \mathrm{L}^{-1}$ sucrose, and $\left.0.5 \mathrm{~g} \cdot \mathrm{L}^{-1} \mathrm{MgSO}_{4} ; \mathrm{pH} 7.0\right)$ on a rotary shaker $(200 \mathrm{rpm})$ at $28{ }^{\circ} \mathrm{C}$ for $16 \mathrm{~h}$. Bacterial cells were collected by centrifugation at $5,000 \mathrm{rpm}$ for $10 \mathrm{~min}$ and suspended in sterilized distilled water. The density of the suspension was adjusted to $\approx 10^{9}$ colony-forming units $/ \mathrm{mL}$.
The inoculation was performed as described by Bliss et al. (1999). Twigs were wounded at three sites by cutting into the cambial area with a scalpel and removing a piece of tissue about $1 \mathrm{~cm}$ long from the stem surface. One drop of prepared bacterial suspension was placed on each wound site, which was wrapped with parafilm to prevent drying. Mock inoculation of the control was performed in a similar manner, but sterilized distilled water was used instead of the bacterial suspension. Two months later, we recorded the maximum diameter of each tumor. The resistance of each germplasm to crown gall was evaluated on the basis of the frequency of tumor occurrence and the tumor size.

In potted trial, when the shoots reached $30 \mathrm{~cm}$ in height, the saplings were transferred to a greenhouse with controlled environment where they were maintained at $28 \pm 3{ }^{\circ} \mathrm{C}$ and a minimum relative humidity of $65 \%$. The saplings were inoculated 1 week later. Bark

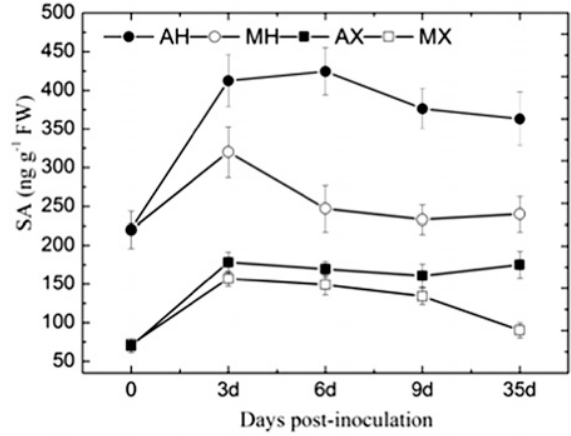

Fig. 1. Chronology of endogenous salicylic acid (SA) levels in Agrobacterium tumefaciensinoculated or mock-inoculated samples. Intact tissues were quantified as 0 period to exclude the wound effect. $\mathrm{AH}=A$. tumefaciensinoculated 'Honggengansutao'; $\mathrm{MH}=$ mock-inoculated 'Honggengansutao'; $\mathrm{AX}=$ $A$. tumefaciens-inoculated 'Xibei 13-1'; MX = mock-inoculated 'Xibei 13-1'. Values are means \pm SE of three independent biological replicates. 


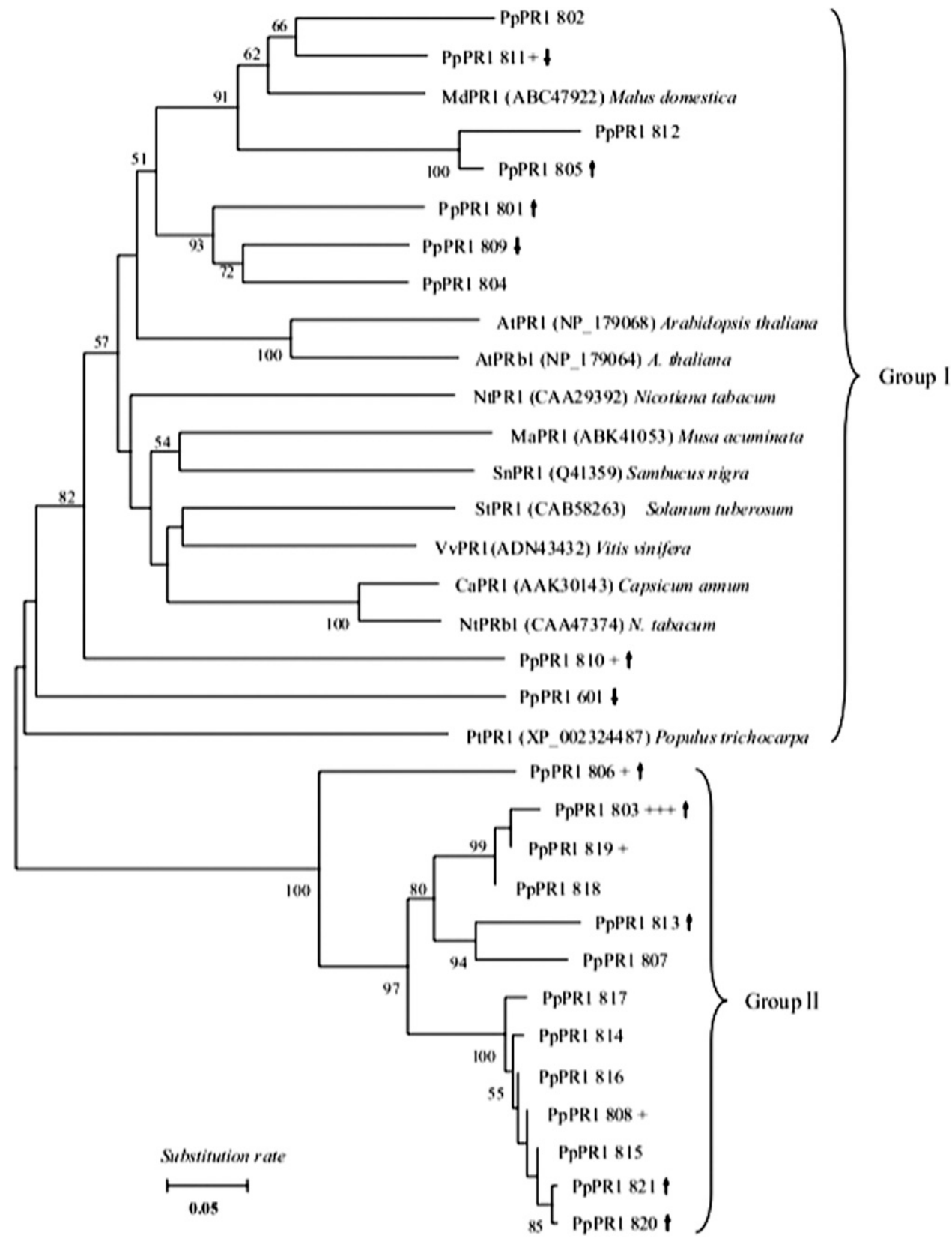

Fig. 2. Phylogenetic tree inferred from PR1 sequences. Bootstrap values are provided only for branches that received bootstrap support of $50 \%$ or more. Six proteins are encoded by two or four identical genes, of which only one is shown in the figure (plus sign indicates the presence of extra copies); ' $\uparrow$ ' and ' $\downarrow$ ' indicate the upregulated and downregulated PPPRI genes, respectively, in response to Agrobacterium tumefaciens infection.

at the inoculation site was harvested at $3,6,9$, and $35 \mathrm{~d}$ after inoculation and immediately frozen in liquid $\mathrm{N}_{2}$ and stored at $-80^{\circ} \mathrm{C}$.

$R N A$ extraction and gene expression. Plant material pooled from five plants was ground with a mortar and pestle under liquid nitrogen into a fine powder. The total RNA was extracted from $200 \mathrm{mg}$ of inoculated or mock tissues using RNeasy Kit (TaKaRa, Tokyo, Japan). RNA samples were treated with RNase-free DNase I (TaKaRa) before the synthesis of cDNA to remove any traces of genomic DNA. The first-strand cDNA was synthesized from $5 \mu \mathrm{g}$ of total RNA using RevertAid $^{\mathrm{TM}}$ Premium First Strand cDNA Synthesis Kit (Fermentas UAB, Burlington, ON, Canada) according to the manufacturer's instructions.
To verify the genes of SAR pathway that were affected by infestation with $A$. tumefaciens, we measured the expression of the $P R 1$ genes and contrasted it against $R P I I$ and TEF2 (Tong et al., 2009) as reference genes (Table 1). The polymerase chain reaction (PCR) reaction was performed in $20 \mu \mathrm{L}$ of reaction mixture containing $1 \mu \mathrm{L}$ cDNA, 10 $\mu \mathrm{L}$ LightCycler ${ }^{\circledR} 480$ SYBR Green Master (Roche, Penzberg, Germany), and $0.5 \mu \mathrm{L}$ $\left(10 \mu \mathrm{mol} \cdot \mathrm{L}^{-1}\right)$ of each primer. The specificity of the individual PCR amplification was confirmed by the melting curve analyses following the final cycle of the PCR. The fold-change in gene expression was calculated using the comparative cycle threshold $\left(C_{\mathrm{t}}\right)$ method $\left(2^{-\Delta C \mathrm{t}}\right)$ (Schmittgen and Livak, 2008).
Genomics database search and in silico analysis of sequences. Gene and protein sequences were retrieved by homology search in the NCBI and the Genome Database for Rosaceae (http://www.rosaceae.org/species/ prunus_persica/genome_v1.0). The peach EST database contained 79,824 entries as of 1 Jan. 2013 (http://www.ncbi.nlm.nih.gov/ genbank/dbest/dbest_summary/) and was not abundant enough. Therefore, the PpPRI gene was confirmed by EST evidence in genus Prunus (http://blast.ncbi.nlm.nih.gov/ Blast.cgi). Multiple alignments of $P p P R I$ genes with orthologs from diverse plant species were conducted using ClustalX (Jeanmougin et al., 1998). The phylogenetic tree was constructed with MEGA6 software using the Neighbor-Joining method with 
1000 bootstrap replications (Tamura et al., 2013).

Quantification of endogenous SA. Endogenous SA was quantified as described by Forcat et al. (2008). Ground samples (150 mg) were weighted and transferred to 2-mL microfuge tubes and each sample was dissolved with $400 \mu \mathrm{L}$ of $10 \%$ methanol containing $1 \%$ acetic acid. Samples were shaken on a rotary shaker at $25^{\circ} \mathrm{C}$ for $10 \mathrm{~min}$ and placed on ice for $30 \mathrm{~min}$. The mixtures were centrifuged at $13,000 g_{\mathrm{n}}$ for $10 \mathrm{~min}$ at $4{ }^{\circ} \mathrm{C}$. The supernatant was carefully removed and the pellet was resuspended with $400 \mu \mathrm{L}$ of $10 \%$ methanol containing $1 \%$ acetic acid. The resuspended mixtures were incubated on ice for $30 \mathrm{~min}$ and centrifuged again. The supernatants resulting from the two extractions were mixed and preserved for later analysis.

Samples were analyzed by liquid chromatography-tandem mass spectrometry (MS) using Agilent 6460B (Agilent Technologies, Santa Clara, CA). Chromatographic separation was carried out on a ZORBAX SB-C18 column $(2.1 \mathrm{~mm} \times 100 \mathrm{~mm} \times 1.8 \mu \mathrm{m})$ (Agilent Technologies). The mobile phase consisted of water-formic acid (99:1) as solvent $\mathrm{A}$ and acetonitrile as solvent $\mathrm{B}$. The gradient profile began at $90 \%$ solvent $\mathrm{A}$ in equal intervals. The flow rate was $0.3 \mathrm{~mL} \cdot \mathrm{min}^{-1}$ and the column temperature was set at $30^{\circ} \mathrm{C}$. The injection volume was $5 \mu \mathrm{L}$. For $\mathrm{MS}$ analyses, nitrogen was used as a drying and nebulizing gas, and nebulizer pressure was set at $275,790 \mathrm{~Pa}$. Gas flow was set at $10 \mathrm{~L} \cdot \mathrm{min}^{-1}$ and temperature was $350{ }^{\circ} \mathrm{C}$. Data were collected and analyzed by MassHunter Workstation B02.06 data acquisition and processing software (Agilent Technologies).

\section{Results}

Verification of the induced resistance by virulent A. tumefaciens invasion in certain peach germplasms. In 2013, a total of 1100 seedlings were screened. All of the inoculation sites that were treated with sterilized distilled water did not result in tumor formation (data not shown). To increase the frequency of inoculation replication, the seedlings were inoculated for the second time at $30 \mathrm{~d}$ after the first inoculation. Except the two accessions 'Honggengansutao' and 'Xibei 13-1', the gall size and/or occurrence frequency in 40 accessions or cultivars did not differ between the two inoculation periods (Table 2).

In 2014, 189 germplasms were screened and 35 germplasms were selected randomly and inoculated for the second and third time at 20 and $40 \mathrm{~d}$ after the first inoculation. The gall sizes between the first and the second, the first and the third, and the second and the third inoculations in 35 germplasms were positively correlated $\left(R^{2}=0.83,0.79\right.$, and 0.91 , respectively; data not shown). Meanwhile, seven were selected from 40 accessions or cultivars that were screened the previous year, and they were inoculated three times successively. The gall sizes of two accessions did not differ between the inoculation periods, and they were smaller than the galls formed after the first inoculation in
2013. The same results were confirmed for the frequency of gall occurrence (Table 2). These data indicated that these two cultivars displayed reinforced resistance to subsequent invasion.

Quantitative analysis of $S A$. Changes in the levels of SA in the host during the infection
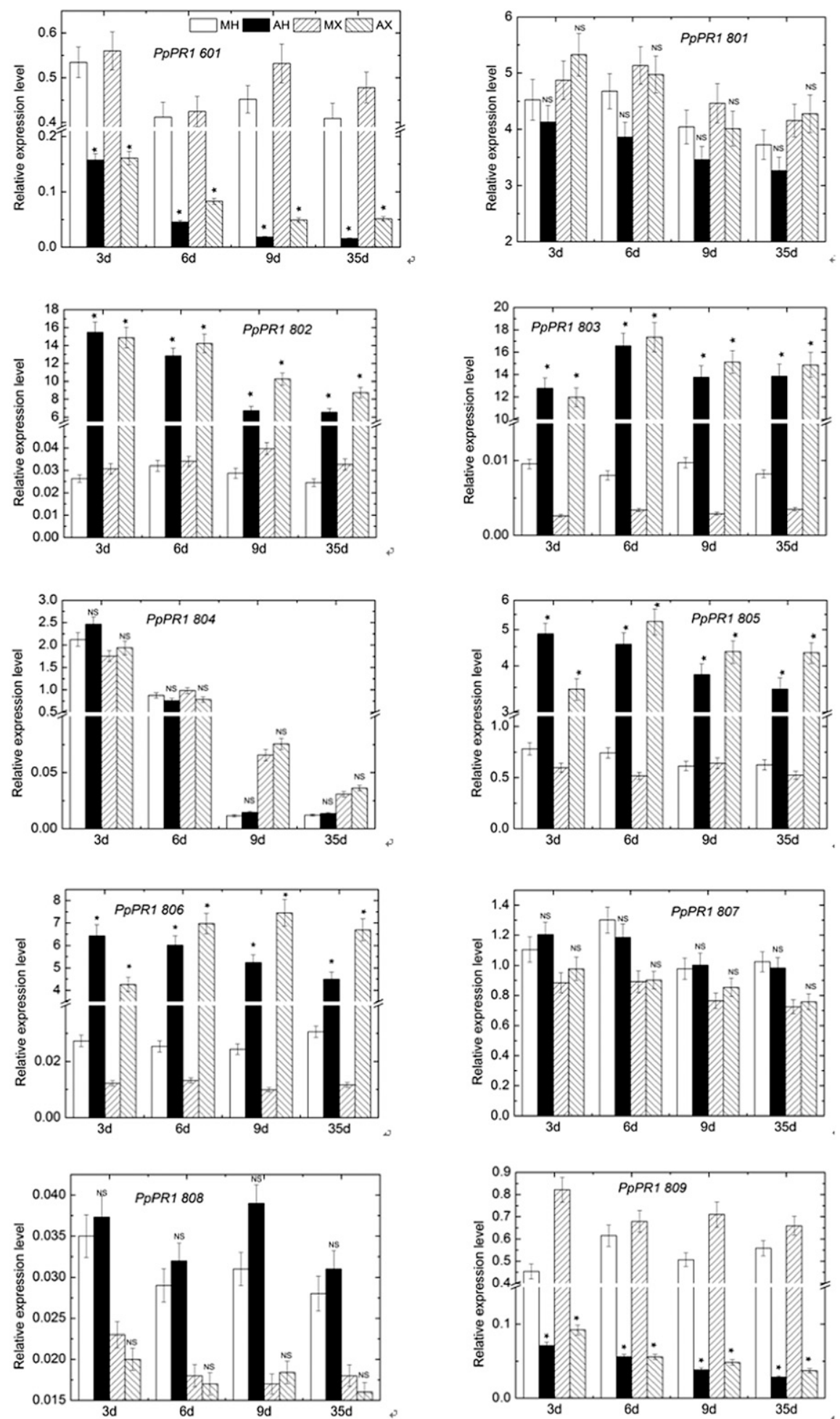

Fig. 3. Transcription expression of $P p P R 1$ genes in response to Agrobacterium tumefaciens infection. The expression levels of each gene were compared against a reference gene and presented as the ratio of the target gene/RPII. $\mathrm{AH}=A$. tumefaciens-inoculated 'Honggengansutao'; $\mathrm{MH}=$ mock-inoculated 'Honggengansutao'; $\mathrm{AX}=$ A. tumefaciens-inoculated 'Xibei 13-1'; MX = mock-inoculated 'Xibei 13-1'. *, NS = Significant and nonsignificant differences, respectively, at $P<0.05$ by Student's $t$ test between the mock- and $A$. tumefaciens-inoculation for the same period. 

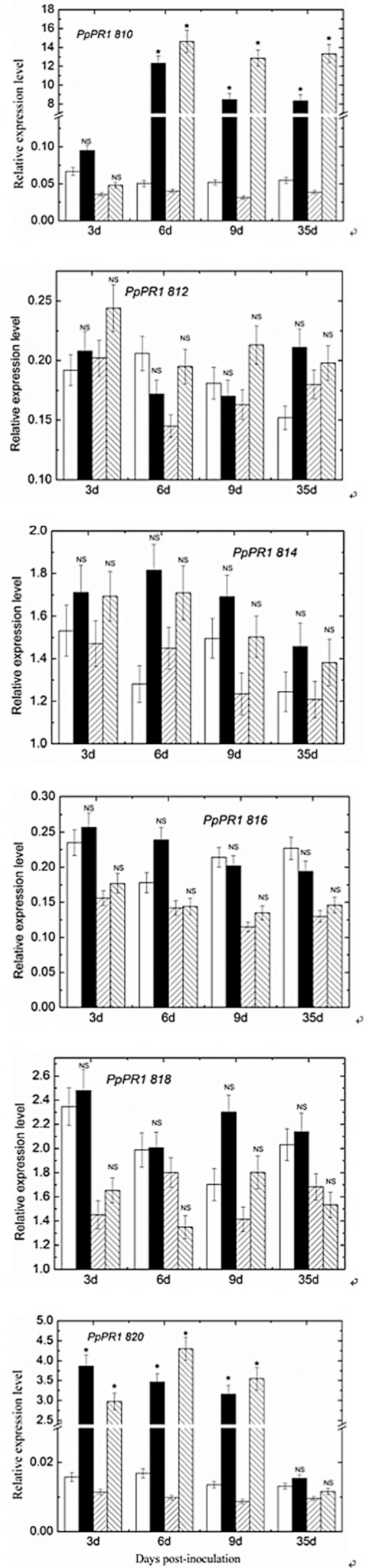

Fig. 3. (Continued)
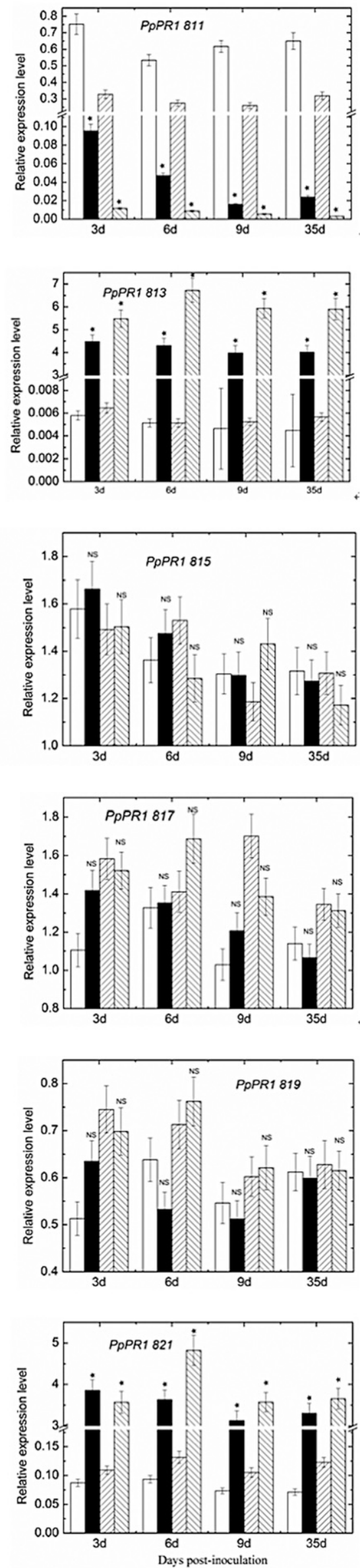

670 level of SA remained similar at 3, 6, 9, and 35 dpi in bacteria-infected 'Xibei 13-1'. The level of SA was much higher in 'Honggengansutao' than in 'Xibei 13-1', and in mock-treated 'Honggengansutao' the levels were higher than in bacteria infected 'Xibei 13-1'.

Identification of the active peach PRI genes from 'Lovell'. An extensive search of the NCBI and the Genome Database for Rosaceae for all possible $P R 1$ genes in peach yielded 30 sequences, excluding nine sequences with no EST evidence. These predicted PR1 protein sequences were aligned and the duplicates with identical sequences were consolidated. Subsequently, a total of 22 nonredundant PR1 proteins were identified from the genome of 'Lovell'. Proposed names of genes were designated according to the location and order on the scaffolds to differentiate each gene. For example, ppa026522m, the first one to appear on scaffold 8, was followed by ppa $027034 \mathrm{~m}$, and these were denoted as PpPR1 801 and PpPR1 802, respectively (duplicates were not counted).

The phylogenetic analysis of PpPR1 proteins and PR1 proteins from other species resulted in two clades. One clade (group I) included nine PpPR1 and 11 orthologs, and the other clade (group II) consisted of 13 paralogs (group II) and received high bootstrap support of $100 \%$ (Fig. 2).

Differential expression of PpPR1 genes in response to $A$. tumefaciens infection. In this study, the expression of PpPRI genes was monitored to indicate the SAR status. We identified 22 candidate PPPRI genes from the peach genome through mining of genomic databases. The transcript profiles of individual $P p P R 1$ genes were analyzed by quantitative reverse transcription-PCR after inoculation with $A$. tumefaciens (Fig. 3). The transcription of PpPR1 802, 803, 805, 806, 810,813, 820, and 821 was induced by bacteria, reaching the maximum levels at 3 or 6 dpi and seven of them maintained these high levels at $35 \mathrm{dpi}$ except $P p P R 1$ 820. However, the transcription of PpPR1 601, 809, and 811 was depressed and maintained at low levels. Transcription of the remaining $10 P p P R 1$ genes did not change and they were constitutively expressed, indicating that, with the exception of PpPR1 804 induced by wounds, these genes had other functions.

\section{Discussion}

In this study, we show that SAR is activated as a consequence of both compatible and incompatible (less compatible) $A$. tumefaciens-peach interactions. The induced resistance protected the plants against the subsequent colonization and development by the same virulent bacterial pathogen, resulting in a lower frequency of tumor occurrence and a smaller gall size (Table 2). Meanwhile, the groove at the site of inoculation became deeper and wider in bacteria-infected branches than in mock-treated branches (Fig. 4). This phenotypical difference may have originated from the PTI and/or ETI responses. The SAR can be triggered by both PTI and/or ETI 

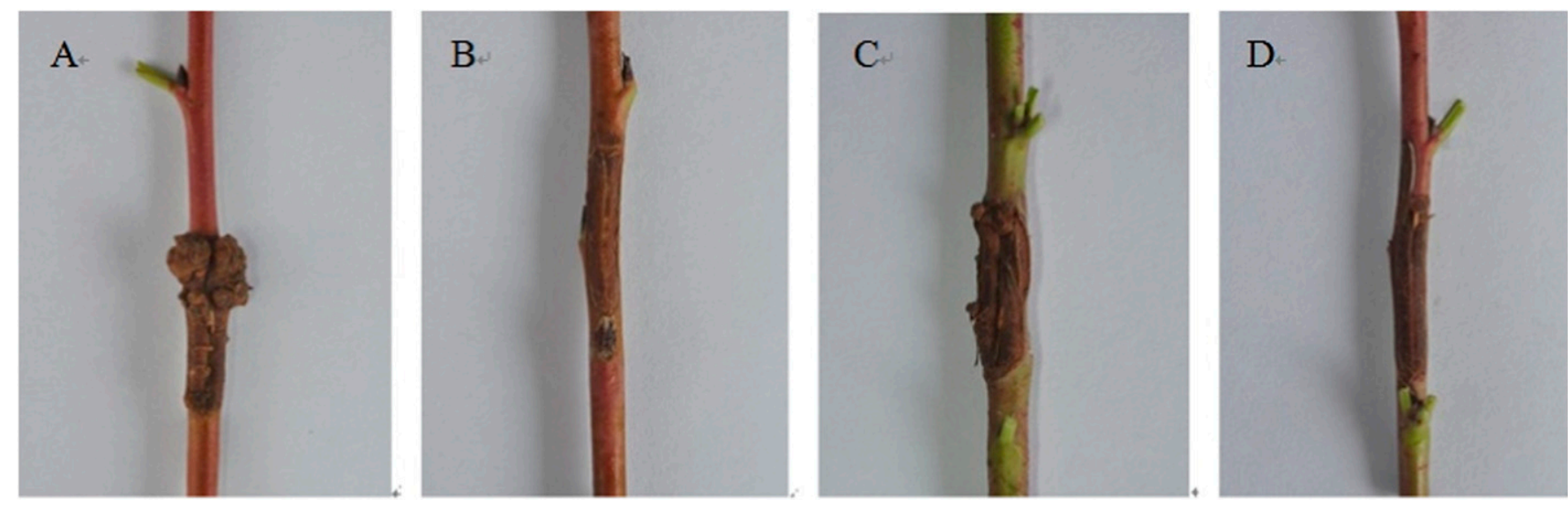

Fig. 4. Symptomatic development on stems of two peach cultivars at 35 d post-inoculation. (A) Agrobacterium tumefaciens-inoculated 'Xibei 13-1'; (B) mockinoculated 'Xibei 13-1'; (C) A. tumefaciens-inoculated 'Honggengansutao'; (D) mock-inoculated 'Honggengansutao'.

(Pieterse et al., 2009). Moreover, this phenotypical difference also existed in non-SAR induced cultivars. We hypothesized that the failure of the SAR induction was due to the SAR pathway rather than bacteria recognition. On the other hand, the SAR induction and the level of protection provided by SAR varies in different plant species or cultivars infected with different pathogens (Bonnet et al., 1996; Cameron et al., 1994; Mishina and Zeier, 2007). When infected with $A$. tumefaciens, the SAR was activated in tobacco, whereas the opposite was reported in Arabidopsis (Anand et al., 2008; Lee et al., 2009). Thus, we speculated that the SAR was induced only in few genotypes/cultivars or the low SAR efficiency was neglected.

The results showed that the levels of SA increased at 3 dpi in infested 'Honggengansutao' and 'Xibei 13-1'. The increase in the levels of SA was manifested as SAR induced instead of ISR induced. Salicylic acid is not only a signal molecule involved in regulation of plant defense, but it also directly affects bacteria (Prithiviraj et al., 2005). The expression of virB1 gene, corresponding to the T-DNA transfer and integration, was inhibited for more than $50 \%$ by $\mathrm{SA}$ at concentration of $2 \mu \mathrm{M}\left(\approx 276 \mathrm{ng} \cdot \mathrm{g}^{-1}\right)$ (Yuan et al., 2007). The levels of SA in intact 'Honggengansutao' and 'Xibei 13-1' cultivars were 220 and $82 \mathrm{ng} \cdot \mathrm{g}^{-1} \mathrm{FW}$, respectively, coinciding with their resistant and susceptible phenotypes. Although the content of SA was less than $2 \mu \mathrm{M}$, it perhaps contributed to the restriction of the $A$. tumefaciens infection.

In the present study, eight $P p P R 1$ genes were upregulated in response to inoculation with $A$. tumefaciens. One of 22 PRI genes identified in Arabidopsis was induced by pathogens (van Loon et al., 2006). Similarly, none of the three identified PRI genes identified in apple were induced by infection with fire blight bacteria (Bonasera et al., 2006). By contrast, the 12 selected PRI genes in rice were all upregulated in response to the infection by blast fungus (Mitsuhara et al., 2008). Thus, induction of $P R 1$ genes in response to pathogens varied among plant species. Moreover, additional three PPPRI genes were downregulated in resistance and susceptibility, exhibiting negative regulation to the bacterial infection. All of the 11 differentially expressed $P p P R 1$ genes were resolved in different clades on the phylogenetic tree (Fig. 2). The expression of $P p P R 1$ 802 was upregulated in response to the inoculation with A. tumefaciens, and it differed from its nearest ortholog in apple (MdPRla) (Bonasera et al., 2006). In contrast, its nearest paralog PpPR1 811 was down regulated, whereas PpPR1820 and its nearest paralog PpPR1 821 were both induced. These results indicated that, despite their sequence similarities, $P R 1$ genes underwent evolutionary divergence as a consequence of functional selection and that several of the genes are associated with the host defense.

Many factors, such as wound and low temperature, have been described to induce the expression of $P R 1$ genes as well as SAR (Mitsuhara et al., 2008; van Loon et al., 2006). The wound, which is essential for $A$. tumefaciens invasion, confused the SAR induced reason. By monitoring the mock treatment concomitantly with the infections, the wound effect was excluded. Studies have shown that SAR was established 24-48 h after pathogen infection (Cameron et al., 1994; Ross, 1961). Once the SAR is activated, plants express a set of $P R$ and other defense genes, and the PR proteins including $P R 1$ and products of other defense genes such as camalexin (Mishina and Zeier, 2007) and coronatine (Spoel and Dong, 2008) provide a multifaceted protection against successive invasions. In this study, the elevated levels of SA and upregulated expression of eight PpPR 1 genes indicated that the SAR has been activated, but obvious morphological changes were apparent in few and small-insize tumors in resistance and in large tumors in susceptible at the inoculation sites(Fig. 4) at $35 \mathrm{dpi}$. The opposing results originated from the unique pathology of $A$. tumefaciens invasion. After $A$. tumefaciens integrated successfully their T-DNA into the host genome, the transformed plant cells proliferated and were irrelevant to pathogens.

We confirmed that SAR was induced by the virulence of $A$. tumefaciens infection in two peach cultivars, but the underlying mechanism of SAR induction needs to be elucidated to facilitate the development of efficient methods for the control of crown gall disease. In addition, although researchers have made many efforts to select crown gall resistant Prunus resources, there are still a limited number of resistant genotypes/cultivars (Bliss et al., 1999; Pierronnet and Salesses, 1996; Zoina and Raio, 1999). Therefore, the inducible crown gall resistance should be considered in evaluation experiments.

\section{Literature Cited}

Anand, A., S.R. Uppalapati, C.M. Ryu, S.N. Allen, L. Kang, Y. Tang, and K.S. Mysore. 2008. Salicylic acid and systemic acquired resistance play a role in attenuating crown gall disease caused by Agrobacterium tumefaciens. Plant Physiol. 146:703-715.

Bliss, F.A., A.A. Almehdi, A.M. Dandekar, P.L. Schuerman, and N. Bellaloui. 1999. Crown gall resistance in accessions of 20 Prunus species. HortScience 34:326-330.

Bonasera, J.M., J.F. Kim, and S.V. Beer. 2006. PR genes of apple: Identification and expression in response to elicitors and inoculation with Erwinia amylovora. BMC Plant Biol. 6:23.

Bonnet, P., E. Bourdon, M. Ponchet, J.P. Blein, and P. Ricci. 1996. Acquired resistance triggered by elicitins in tobacco and other plants. Eur. J. Plant Pathol. 102:181-192.

Cameron, R.K., R.A. Dixon, and C.J. Lamb. 1994 Biologically induced systemic acquired resistance in Arabidopsis thaliana. Plant J. 5:715-725.

Choi, Y.J., H.K. Yun, K.S. Park, J.H. Noh, Y.Y Heo, S.H. Kim, D.W. Kim, and H.J. Lee. 2010. Transcriptional profiling of ESTs responsive to Rhizobium vitis from 'Tamnara' grapevines (Vitis sp.). J. Plant Physiol. 167:1084-1092.

Durrant, W.E. and X. Dong. 2004. Systemic acquired resistance. Annu. Rev. Phytopathol. 42:185-209.

Escobar, M.A. and A.M. Dandekar. 2003. Agrobacterium tumefaciens as an agent of disease. Trends Plant Sci. 8:380-386.

Forcat, S., M.H. Bennett, J.W. Mansfield, and M.R. Grant. 2008. A rapid and robust method for simultaneously measuring changes in the phytohormones ABA, JA and SA in plants following biotic and abiotic stress. Plant Methods 4:16.

Govrin, E.M. and A. Levine. 2002. Infection of Arabidopsis with a necrotrophic pathogen, Botrytis cinerea, elicits various defense responses but does not induce systemic acquired resistance (SAR). Plant Mol. Biol. 48:267-276. 
Jeanmougin, F., J.D. Thompson, M. Gouy, D.G. Higgins, and T.J. Gibson. 1998. Multiple sequence alignment with Clustal X. Trends Biochem. Sci. 23:403-405.

Jones, J.D. and J.L. Dangl. 2006. The plant immune system. Nature 444:323-329.

Lee, C.W., M. Efetova, J.C. Engelmann, R. Kramell, C. Wasternack, J. Ludwig-Müller, R. Hedrich, and R. Deeken. 2009. Agrobacterium tumefaciens promotes tumor induction by modulating pathogen defense in Arabidopsis thaliana. Plant Cell 21:2948-2962.

Loake, G. and M. Grant. 2007. Salicylic acid in plant defence-the players and protagonists. Curr. Opin. Plant Biol. 10:466-472.

Mishina, T.E. and J. Zeier. 2007. Pathogenassociated molecular pattern recognition rather than development of tissue necrosis contributes to bacterial induction of systemic acquired resistance in Arabidopsis. Plant J. 50:500-513.

Mitsuhara, I., T. Iwai, S. Seo, Y. Yanagawa, H. Kawahigasi, S. Hirose, Y. Ohkawa, and Y. Ohashi. 2008. Characteristic expression of twelve rice $P R 1$ family genes in response to pathogen infection, wounding, and defenserelated signal compounds (121/180). Mol. Genet. Genomics 279:415-427.

Pierronnet, A. and G. Salesses. 1996. Behaviour of Prunus cultivars and hybrids towards Agrobacterium tumefaciens estimated from hardwood cuttings. Agronomie 16:247-256.

Pieterse, C.M., A. Leon-Reyes, S. Van der Ent, and S.C. Van Wees. 2009. Networking by small-molecule hormones in plant immunity. Nat. Chem. Biol. 5:308-316.

Pitzschke, A. and H. Hirt. 2010. New insights into an old story: Agrobacterium-induced tumour formation in plants by plant transformation. EMBO J. 29:1021-1032.

Prithiviraj, B., H.P. Bais, T. Weir, B. Suresh, E.H. Najarro, B.V. Dayakar, H.P. Schweizer, and J.M. Vivanco. 2005. Down regulation of virulence factors of Pseudomonas aeruginosa by salicylic acid attenuates its virulence on Arabidopsis thaliana and Caenorhabditis elegans. Infect. Immun. 73:5319-5328.

Ross, A.F. 1961. Systemic acquired resistance induced by localized virus infections in plants. Virology 14:340-358.

Schmittgen, T.D. and K.J. Livak. 2008. Analyzing real-time PCR data by the comparative $C_{T}$ method. Nat. Protoc. 3:1101-1108.

Sherif, S., G. Paliyath, and S. Jayasankar. 2012. Molecular characterization of peach PR genes and their induction kinetics in response to bacterial infection and signaling molecules. Plant Cell Rpt. 31:697-711.

Spoel, S.H. and X. Dong. 2008. Making sense of hormone crosstalk during plant immune responses. Cell Host Microbe. 3:348-351.

Tamura, K., G. Stecher, D. Peterson, A. Filipski, and S. Kumar. 2013. MEGA6: Molecular evolutionary genetics analysis version 6.0. Mol. Biol. Evol. 30:2725-2729.

Tong, Z., Z. Gao, F. Wang, J. Zhou, and Z. Zhang. 2009. Selection of reliable reference genes for gene expression studies in peach using realtime PCR. BMC Mol. Biol. 10:71.

Vallad, G.E. and R.M. Goodman. 2004. Systemic acquired resistance and induced systemic resistance in conventional agriculture. Crop Sci. 44:1920-1934.

van Loon, L.C., P.A. Bakker, and C.M. Pieterse. 1998. Systemic resistance induced by rhizosphere bacteria. Annu. Rev. Phytopathol. 36:453-483.

van Loon, L.C., M. Rep, and C.M. Pieterse. 2006. Significance of inducible defense-related proteins in infected plants. Annu. Rev. Phytopathol. 44:135-162.

van Loon, L.C. and E.A. van Strien. 1999. The families of pathogenesis-related proteins, their activities, and comparative analysis of PR-1 type proteins. Physiol. Mol. Plant Pathol. 55:85-97.

Wu, Y., G. Yi, X. Peng, B. Huang, E. Liu, and J. Zhang. 2013. Systemic acquired resistance in Cavendish banana induced by infection with an incompatible strain of Fusarium oxysporum f. sp. cubense. J. Plant Physiol. 170:1039-1046.

Yuan, Z.C., M.P. Edlind, P. Liu, P. Saenkham, L.M. Banta, A.A. Wise, E. Ronzone, A.N. Binns, K. Kerr, and E.W. Nester. 2007. The plant signal salicylic acid shuts down expression of the vir regulon and activates quormone-quenching genes in Agrobacterium. Proc. Natl Acad. Sci. USA 104:11790-11795.

Zoina, A. and A. Raio. 1999. Susceptibility of some peach rootstocks to crown gall. J. Plant Pathol. 81:181-187. 